\title{
Treadmill and wheel exercise alleviate lipopolysaccharide- induced short-term memory impairment by enhancing neuronal maturation in rats
}

\author{
SUNG-EUN KIM ${ }^{1}$, IL-GYU KO ${ }^{1,2}$, CHANG-YOUL PARK ${ }^{1}$, MAL-SOON SHIN ${ }^{1}$, \\ CHANG-JU KIM ${ }^{1}$ and YONG-SEOK JEE ${ }^{2}$ \\ ${ }^{1}$ Department of Physiology, College of Medicine, Kyung Hee University, Seoul 130-701; \\ ${ }^{2}$ Department of Physical Activity Design, College of Science, Hanseo University, Seosan 356-706, Republic of Korea
}

Received August 31, 2012; Accepted October 18, 2012

DOI: $10.3892 / \mathrm{mmr} .2012 .1160$

\begin{abstract}
Lipopolysaccharide (LPS) is an endotoxin derived from Gram-negative bacteria, which induces brain inflammation. LPS-induced brain inflammation deteriorates hippocampus-dependent cognitive deficits. In the present study, we investigated the effects of forced treadmill exercise and voluntary wheel exercise on short-term memory in relation to neuronal maturation in LPS-induced brain inflammation of rats. Brain inflammation in rats was induced by an injection of LPS into the cerebral ventricle. Short-term memory was evaluated using a step-down avoidance task. Cell proliferation in the hippocampal dentate gyrus was determined by 5-bromo-2'-deoxyuridine (BrdU), a marker of new cells, immunohistochemistry. Western blot analysis for the determination of doublecortin (DCX), a marker of immature neurons and neuronal nuclear antigen (NeuN), a marker of mature neurons, was performed. In the present study, LPS-induced brain inflammation impaired short-term memory by increasing DCX expression and suppressing NeuN expression. These results suggest that LPS-induced brain inflammation disturbs neuronal maturation. The number of BrdU-positive cells in the hippocampal dentate gyrus was increased by LPS injection. This increase in the number of BrdU-positive cells can be ascribed to the increase in the number of of immature neurons following LPS injection. On the other hand, forced treadmill exercise and voluntary wheel exercise improved brain inflammation-induced short-term memory impairment by suppressing DCX expression and increasing NeuN expression, enhancing neuronal maturation. Forced treadmill exercise and voluntary wheel exercise showed similar efficacy. From these results, it can be inferred
\end{abstract}

Correspondence to: Professor Yong-Seok Jee, Department of Physical Activity Design, College of Science, Hanseo University, 360 Daegok-ri, Haemi-myun, Seosan 356-706, Republic of Korea.

E-mail: jeeys@hanseo.ac.kr

Key words: lipopolysaccharide, brain inflammation, hippocampus, short-term memory, doublecortin, neuronal nuclear antigen that forced treadmill exercise and voluntary wheel exercise may improve memory function deteriorated by brain inflammation.

\section{Introduction}

Lipopolysaccharide (LPS) is an endotoxin derived from Gram-negative bacteria and it is a potent stimulus activating glial cells in the brain. LPS-induced microglial activation affects the survival of newly formed hippocampal neurons (1). The survival of hippocampal progenitors has been shown to decrease following co-culture with microglial cells activated by LPS (2), substantially preventing neuronal differentiation and significantly increasing glial differentiation (3). An LPS injection into the lateral ventricle has been shown to cause deficits in the ability of spatial learning as regards the Morris water maze test (4). Chronic neuroinflammation is a hallmark of several neurological disorders associated with cognitive loss and LPS-induced brain inflammation deteriorates hippocampusdependent cognitive deficits (5).

Physical exercise has been demonstrated to exert a protective effect against various brain disorders (6-8). In particular, physical exercise has been shown to increase neurogenesis in the hippocampal dentate gyrus and newly generated neurons in the hippocampal dentate gyrus improve learning ability and memory function $(7,9,10)$.

Neurogenesis in the hippocampal dentate gyrus occurs throughout post-natal life, including adult life and is influenced by the environment and behavior (10-12). Adult hippocampal neurogenesis is considered to be composed of several developmental stages: proliferation, differentiation, migration, targeting and synaptic integration and various characteristic neuronal markers are expressed during the specific stages (13-15).

Among these neuronal markers, doublecortin (DCX) is a brain-specific microtubule-associated protein and is considered as the immature neuronal marker. DCX promotes microtubule polymerization and is present in migrating neuroblasts and young neurons (16). DCX is also present in the tips of neurites of non-migratory immature neurons, indicating that it plays a role in the growth of neuronal processes, downstream of directional or guidance signals. Thus, DCX is expressed during neurogenesis by mitotic and early post-mitotic neurons $(15,17)$. 
On the other hand, the neuronal nuclear antigen (NeuN) can be used as a marker of newly generated neuronal cells. NeuN is considered as a specific marker of neurons, as it is not expressed in non-neuronal cells (18). In addition, it does not stain the nuclei of immature nerve cells until they achieve a stage of development that approaches mature function. Therefore, NeuN is regarded as a useful marker of neuronal maturation (19).

As mentioned above, physical exercise increases neurogenesis in the hippocampal dentate gyrus, resulting in the improvement or maintenance of learning ability and memory function $(7,9,10)$. However, the memory-enhancing effects of exercise in association with neuronal maturation have not yet been evaluated. In the present study, we investigated the effects of forced treadmill exercise and voluntary wheel exercise on the neuronal maturation in rats with LPS-induced brain inflammation. Brain inflammation in rats was induced by an injection of LPS into the cerebral ventricle. Short-term memory was evaluated using a step-down avoidance task. Cell proliferation was determined by bromo-2'-deoxyuridine (BrdU) immunohistochemistry. Western blot analysis for the determination of DCX and NeuN expression in the hippocampus was performed in regards to neuronal maturation.

\section{Materials and methods}

Animals. Six-week-old male Sprague-Dawley rats weighing $200 \pm 10 \mathrm{~g}(\mathrm{n}=60)$ were used for this experiment. The experimental procedures were performed in accordance with the animal care guidelines of the National Institutes of Health (NIH) and the Korean Academy of Medical Sciences. The rats were housed under controlled temperature $\left(20 \pm 2^{\circ} \mathrm{C}\right)$ and lighting (07:00 to 19:00 h) conditions with food and water available ad libitum. The rats were randomly divided into 4 groups ( $n=15$ each): the control group, the LPS injection group, the LPS injection and treadmill exercise group and the LPS injection and wheel exercise group. All rats received $50 \mathrm{mg} / \mathrm{kg}$ (BrdU; Sigma Chemical Co., St. Louis, MO, USA) intraperitoneally (i.p.) once a day 30 min prior to the onset of treadmill and wheel exercise for the first 4 days.

Induction of brain inflammation. Brain inflammation was induced by an injection of LPS (055:B5; Sigma Chemical Co.) into the cerebral ventricle, as previously described (20). Briefly, the rats were anesthetized with Zoletil $50^{\circledR}(10 \mathrm{mg} / \mathrm{kg}$ i.p.; Virbac Laboratories, Carros, France) and placed in a stereotaxic frame. Through a hole drilled into the skull, a 26-gauge needle was implanted into the cerebral ventricle at the following coordinates: $1.2 \mathrm{~mm}$ lateral to the midline, $0.9 \mathrm{~mm}$ anterior to the coronal suture and at a depth of $3.3 \mathrm{~mm}$ from the surface of the brain. LPS was dissolved in artificial cerebrospinal fluid and $50 \mu \mathrm{g}$ of LPS in $7 \mu \mathrm{l}$ was infused for over $3 \mathrm{~min}$. The needle remained in place for an additional 3 min after the infusion and was then gradually withdrawn.

Exercise protocols. Prior to the onset of exercise, the daily distance for each rat on the wheel was calculated for 1 week, using an extra number of rats $(n=10)$. The exercise load of treadmill running was adjusted to the exercise load of wheel running. Treadmill and wheel exercise began 1 day following LPS injection.
The rats in the treadmill exercise group were forced to run on a motorized treadmill for 30 min once a day for 6 weeks. The exercise load consisted of running at a speed of $5 \mathrm{~m} / \mathrm{min}$ for the first $5 \mathrm{~min}, 8 \mathrm{~m} / \mathrm{min}$ for the next $5 \mathrm{~min}$ and $10 \mathrm{~m} / \mathrm{min}$ for the last $20 \mathrm{~min}$, with $0^{\circ}$ inclination.

The rats in the wheel exercise group were individually placed in cages equipped with a running wheel (diameter, $20 \mathrm{~cm}$; width, $9 \mathrm{~cm}$ ). A magnet attached to a running wheel triggered a magnetic reed switch that provided input to an electrical counter. The number of revolutions was recorded and wheel revolutions were counted irrespective of the direction of the wheel. The running distance was calculated using the number of wheel revolutions. The rats in the non-exercise groups were left in the treadmill without running for the same period of time as the rats in the treadmill exercise group.

Step-down avoidance task. The latency time of the step-down avoidance task was determined in order to evaluate short-term memory, as previously described (7). Briefly, the rats were trained in a step-down avoidance task 38 days after beginning the treadmill and wheel exercise. Twenty four hours after training, the latency time (sec) in each group was measured.

The rats were placed on a $7 \times 25-\mathrm{cm}$ platform, with a height of $2.5 \mathrm{~cm}$. The platform faced a $42 \times 25-\mathrm{cm}$ grid of parallel stainless steel bars, $0.1 \mathrm{~cm}$ in caliber, spaced $1 \mathrm{~cm}$ apart. In the training sessions, the animals received a $0.5 \mathrm{~mA}$ scrambled foot shock for 2 sec immediately upon stepping down. The time interval that elapsed between the rats stepping down and placing all four paws on the grid was defined as the latency time. A latency time $>300 \mathrm{sec}$ was counted as $300 \mathrm{sec}$.

Tissue preparation. The rats were sacrificed immediately after determining the latency time of the step-down avoidance task. All rats were anesthetized using Zoletil 50 (10 mg/kg, i.p.; Virbac Laboratories), transcardially perfused with $50 \mathrm{mM}$ phosphate-buffered saline (PBS) and fixed with a freshly-prepared solution consisting of $4 \%$ paraformaldehyde in $100 \mathrm{mM}$ phosphate buffer (PB, $\mathrm{pH}$ 7.4). The brains were dissected and post-fixed in the same fixative method overnight and transferred to a $30 \%$ sucrose solution for cryoprotection. Coronal sections (49 $\mu$ m-thick) were made using a freezing microtome (Leica, Nussloch, Germany). On average, 5 slice sections in the hippocampus were collected from each rat. The sections obtained 2.5-2.7 $\mathrm{mm}$ posterior to the bregma were used for BrdU immunohistochemistry.

Immunohistochemistry for BrdU. In order to detect newly generated cells in the hippocampal dentate gyrus, BrdU-specific immunohistochemistry was performed, as previously described (7). Briefly, the sections were first permeabilized by incubation in $0.5 \%$ Triton X-100 in PBS for $20 \mathrm{~min}$, then pretreated in $50 \%$ formamide-2X standard saline citrate (SSC) at $65^{\circ} \mathrm{C}$ for $2 \mathrm{~h}$, denatured in $2 \mathrm{~N} \mathrm{HCl}$ at $37^{\circ} \mathrm{C}$ for $30 \mathrm{~min}$ and rinsed twice in $100 \mathrm{mM}$ sodium borate $(\mathrm{pH} 8.5)$. Afterwards, the sections were incubated overnight at $4^{\circ} \mathrm{C}$ with a BrdU-specific mouse monoclonal antibody (1:600; Roche Diagnostics $\mathrm{GmbH}$, Mannheim, Germany). The sections were then washed 3 times with PBS and incubated with a biotinylated mouse secondary antibody (1:200; Vector Laboratories, Burlingame, CA, USA) for $1 \mathrm{~h}$. The sections were then incubated for a further $1 \mathrm{~h}$ with 
an avidin-peroxidase complex (1:100; Vector Laboratories). For visualization, the sections were incubated in $50 \mathrm{mM}$ Tris- $\mathrm{HCl}$ (pH 7.6) containing 0.03\% 3,3'-diaminobenzidine tetrahydrochloride (DAB; Sigma Chemical Co.), $40 \mathrm{mg} / \mathrm{ml}$ nickel chloride and $0.03 \%$ hydrogen peroxide for $5 \mathrm{~min}$. The sections were then washed 3 times with PBS and mounted onto gelatin-coated slides. The slides were air-dried overnight at room temperature and the coverslips were mounted using Permount ${ }^{\circledR}$. The numbers of BrdU-positive cells in the dentate gyrus were counted hemilaterally under a light microscope (Olympus) and they were expressed as the numbers of cells per $\mathrm{mm}^{2}$ in the dentate gyrus. The area of the dentate gyrus was measured by the Image-Pro ${ }^{\circledR}$ Plus image analysis system (Media Cybernetics Inc., Silver Spring, MD, USA).

Western blot analysis. Western blot analysis was performed as previously described (7). The hippocampal tissues were collected and were then immediately frozen at $-70^{\circ} \mathrm{C}$. The hippocampal tissues were homogenized on ice and lysed in a lysis buffer containing $50 \mathrm{mM}$ HEPES (pH 7.5), $150 \mathrm{mM} \mathrm{NaCl}$, $10 \%$ glycerol, $1 \%$ Triton X-100, 1 mM PMSF, 1 mM EGTA, $1.5 \mathrm{mM} \mathrm{MgCl} \mathrm{M}_{2} \cdot 6 \mathrm{H}_{2} \mathrm{O}, 1 \mathrm{mM}$ sodium orthovanadate and $100 \mathrm{mM}$ sodium fluoride. Protein content was measured using a Bio-Rad colorimetric protein assay kit (Bio-Rad, Hercules, CA, USA).

Protein $(30 \mu \mathrm{g})$ was separated on SDS-polyacrylamide gels and transferred onto a nitrocellulose membrane. The mouse $\beta$-actin antibody (1:1,000; Santa Cruz Biotechnology Inc., Santa Cruz, CA, USA), mouse anti-NeuN antibody (1:500; Chemicon International) and goat anti-DCX antibody (1:500; Santa Cruz Biotechnology Inc.) were used as the primary antibodies. Horseradish peroxidase-conjugated anti-mouse antibodies for $\beta$-actin and NeuN (1:2,000; Vector Laboratories) and horseradish peroxidase-conjugated anti-goat antibody for DCX (1:5,000; Santa Cruz Biotechnology Inc.) were used as the secondary antibodies.

The experiments were performed under normal laboratory conditions and at room temperature, except for the transferred membranes, which were performed at $4^{\circ} \mathrm{C}$ with a cold pack and a pre-chilled buffer. Band detection was performed using the enhanced chemiluminescence (ECL) detection kit (Santa Cruz Biotechnology Inc.). In order to compare the relative protein expression levels, the detected bands were calculated densitometrically using Molecular Analyst ${ }^{\mathrm{TM}}$, version 1.4.1 (Bio-Rad).

Data analysis. Statistical analysis was performed using IBM PSS software (version 20.0; IBM Corp., Armonk, NY, USA). For the comparison among the groups, one-way ANOVA and Duncan's post hoc test were performed. All values were expressed as the means \pm standard error of the mean (SEM). A $\mathrm{P}$-value $<0.05$ was considered to indicate a statistically significant difference.

\section{Results}

Daily running distance. The daily running distance was $265.00 \pm 0.00 \mathrm{~m}$ in the treadmill exercise group and $253.35 \pm 9.39 \mathrm{~m}$ in the wheel exercise group. There was little difference in the daily running distance between treadmill and wheel exercise.

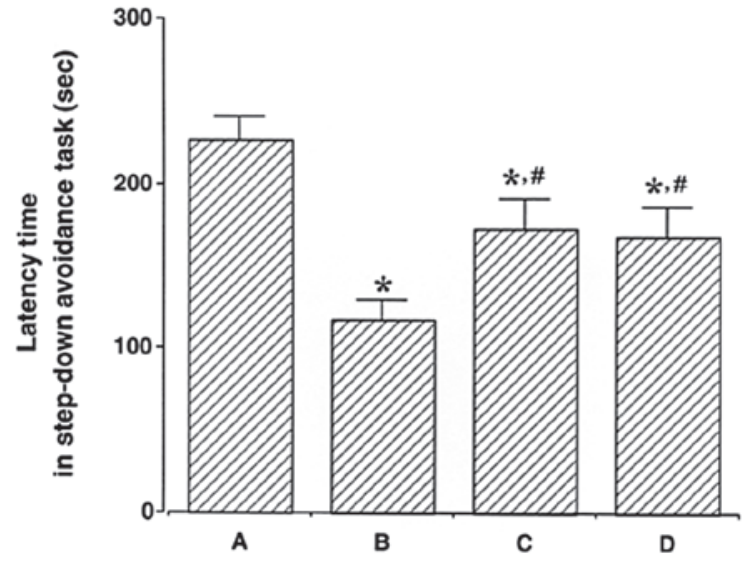

Figure 1. Effects of forced treadmill exercise and voluntary wheel exercise on short-term memory. (A) Control group, (B) LPS injection group, (C) LPS injection and treadmill exercise group and (D) LPS injection and wheel exercise group. Data are presented as the means \pm standard error of the mean (SEM). ${ }^{*} \mathrm{P}<0.05$ compared to the control group; ${ }^{~} \mathrm{P}<0.05$ compared to the LPS injection group.

Short-term memory. In order to evaluate the effects of forced treadmill exercise and voluntary wheel exercise on short-term memory, a step-down avoidance task was performed. The results of the step-down avoidance task are presented in Fig. 1. The latency time was $227.25 \pm 13.70 \mathrm{sec}$ in the control group, $117.75 \pm 12.15 \mathrm{sec}$ in the LPS injection group, $172.30 \pm 18.05 \mathrm{sec}$ in the LPS injection and treadmill exercise group and $167.33 \pm 18.53 \mathrm{sec}$ in the LPS injection and wheel exercise group.

The latency time in the LPS injection group was conspicuously shorter than that in the control group $(\mathrm{P}<0.05)$. On the other hand, treadmill and wheel exercise significantly increased the latency time in the LPS-injected rats $(\mathrm{P}<0.05)$. The effects of treadmill and wheel exercise on short-term memory were similar. These results suggest that forced treadmill exercise and voluntary wheel exercise significantly alleviated LPS-induced short-term memory impairment.

Cell proliferation in the hippocampal dentate gyrus. Photomicrographs of BrdU-positive cells in the hippocampal dentate gyrus are presented in Fig. 2. The number of BrdU-positive cells was $112.19 \pm 4.92 / \mathrm{mm}^{2}$ in the control group, $225.05 \pm 8.57 / \mathrm{mm}^{2}$ in the LPS injection group, $149.08 \pm 4.19 / \mathrm{mm}^{2}$ in the LPS injection and treadmill exercise group and $155.43 \pm 10.26 / \mathrm{mm}^{2}$ in the LPS injection and wheel exercise group.

The number of BrdU-positive cells in the hippocampal dentate gyrus was remarkably increased following LPS injection $(\mathrm{P}<0.05)$; however, treadmill and wheel exercise significantly reduced the number of BrdU-positive cells in the LPS-injected rats $(\mathrm{P}<0.05)$. These results demonstrated that the cell proliferation in the hippocampal dentate gyrus was enhanced by LPS-induced brain inflammation, while forced treadmill exercise and voluntary wheel exercise suppressed cell proliferation in the hippocampal dentate gyrus. The effects of treadmill and wheel exercise on cell proliferation were similar. 

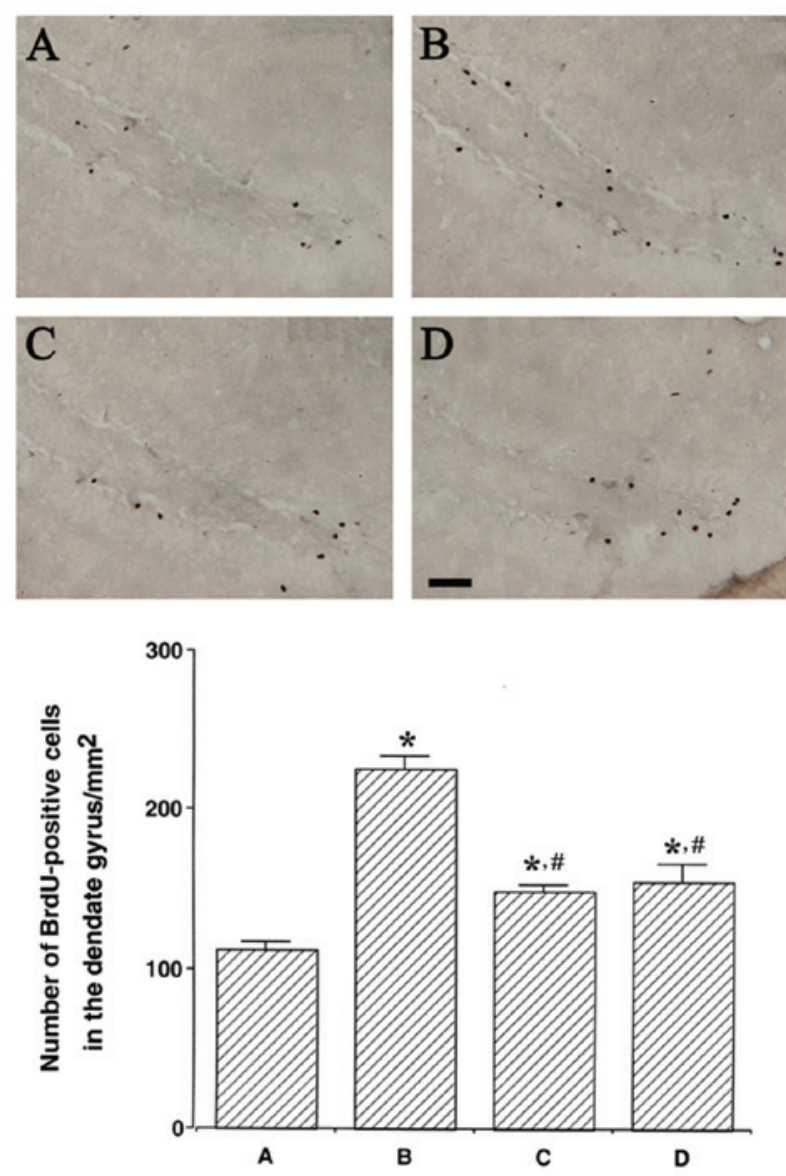

Figure 2. Effects of forced treadmill exercise and voluntary wheel exercise on cell proliferation. Upper panel: Photomicrographs of 5-bromo-2'-deoxyuridine (BrdU)-positive cells in the dentate gyrus. The black scale bar represents $50 \mu \mathrm{m}$. Lower panel: The number of BrdU-positive cells in the dentate gyrus of each group. (A) Control group, (B) LPS injection group, (C) LPS injection and treadmill exercise group and (D) LPS injection and wheel exercise group. Data are presented as the means \pm standard error of the mean $(\mathrm{SEM}) .{ }^{*} \mathrm{P}<0.05$ compared to the control group; ${ }^{"} \mathrm{P}<0.05$ compared to the LPS injection group.
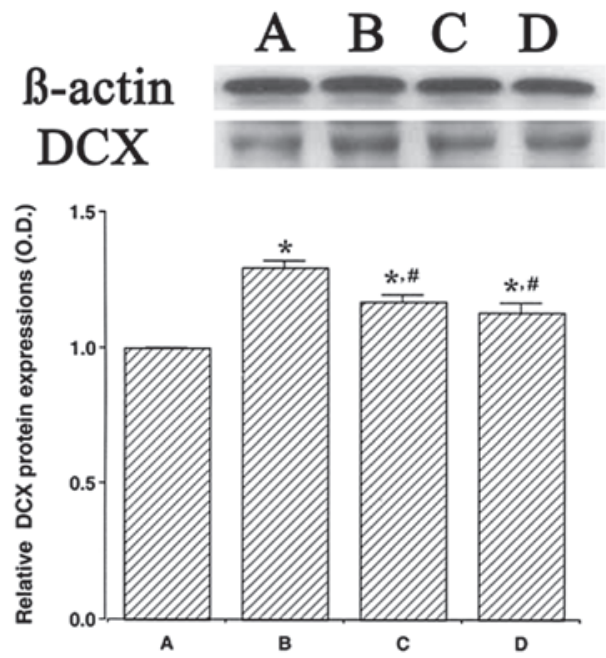

Figure 3. Effects of forced treadmill exercise and voluntary wheel exercise on the expression of DCX protein in each group. (A) Control group, (B) LPS injection group, (C) LPS injection and treadmill exercise group and (D) LPS injection and wheel exercise group. Data are presented as the means \pm standard error of the mean (SEM). ${ }^{*} \mathrm{P}<0.05$ compared to the control group; ${ }^{*} \mathrm{P}<0.05 \mathrm{com}$ pared to the LPS injection group.

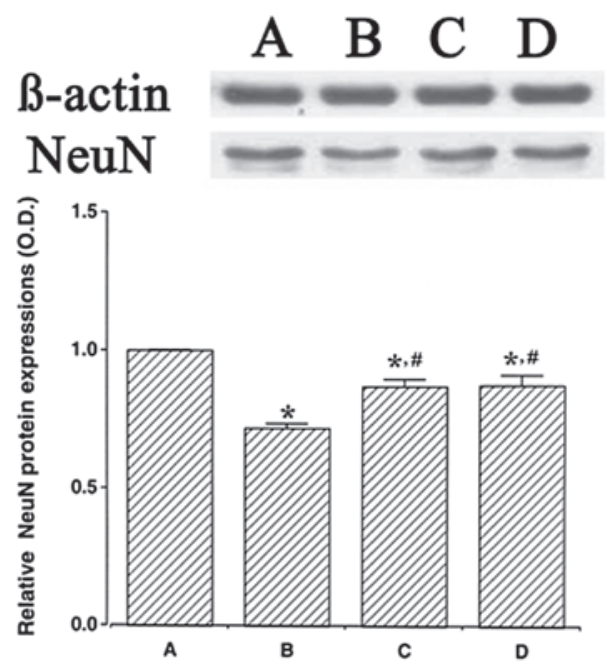

Figure 4. Effects of forced treadmill exercise and voluntary wheel exercise on the expression of the neuronal nuclear antigen $(\mathrm{NeuN})$ protein in each group. (A) Control group, (B) lipopolysaccharide (LPS) injection group, (C) LPS injection and treadmill exercise group, and (D) LPS injection and wheel exercise group. Data are presented as the means \pm standard error of the mean (SEM). ${ }^{*} \mathrm{P}<0.05$ compared to the control group. ${ }^{*} \mathrm{P}<0.05$ compared to the LPS injection group.

Expression of DCX protein in the hippocampus. We determined the relative expression levels of DCX in the hippocampus (Fig. 3). When the level of DCX (45 kDa) in the control group was set at 1.00 , the level of DCX was $1.30 \pm 0.02$ in the LPS injection group, $1.17 \pm 0.03$ in the LPS injection and treadmill exercise group and $1.13 \pm 0.03$ in the LPS injection and wheel exercise group.

The level of DCX protein in the LPS injection group was significantly increased in comparison with that in the control group $(\mathrm{P}<0.05)$. However, treadmill and wheel exercise significantly reduced the expression of DCX protein in the LPS-injected rats $(\mathrm{P}<0.05)$. These results suggest that LPS-induced brain inflammation increases the generation of immature neurons. However, forced treadmill exercise and voluntary wheel exercise suppressed the generation of immature neurons. The effects of treadmill exercise and wheel exercise on the DCX expression were similar.

Expression of NeuN protein in the hippocampus. We determined the relative expression levels of NeuN in the hippocampus (Fig. 4). When the level of NeuN (46-48 kDa) in the control group was set at 1.00 , the level of NeuN was $0.72 \pm 0.02$ in the LPS injection group, $0.87 \pm 0.02$ in the LPS injection and treadmill exercise group and $0.87 \pm 0.03$ in the LPS injection and wheel exercise group.

The expression of NeuN protein in the LPS injection group was significantly decreased in comparison to the control group $(\mathrm{P}<0.05)$. However, treadmill exercise and wheel exercise significantly enhanced the expression of $\mathrm{NeuN}$ protein $(\mathrm{P}<0.05)$. These results suggest that LPS-induced brain inflammation disturbed the maturation of neuronal cells. However, forced treadmill exercise and voluntary wheel exercise enhanced the generation of mature neuronal cells. The effects of treadmill exercise and wheel exercise on NeuN expression were similar. 


\section{Discussion}

Belarbi et al (5) reported that brain inflammation induced by LPS impaired learning ability and memory functions. Similarly, our results revealed that LPS-induced brain inflammation deteriorated short-term memory in rats. However, forced treadmill exercise and voluntary wheel exercise improved short-term memory incapacitated by brain inflammation, regardless of the type of exercise (Fig. 1).

Exercise improves learning ability and memory function by increasing neurogenesis in the hippocampus (21). Neurogenesis induced by exercise is reported to be necessary in hippocampal-dependent learning, such as spatial navigation learning or exploration in the elevated plus maze $(22,23)$. Neurogenesis is also influenced by pathological conditions affecting the brain. Ekdahl et al (1) reported that brain inflammation impaired basal hippocampal neurogenesis by microglial activation. As a result, they suggested that this suppression of hippocampal neurogenesis might contribute to cognitive dysfunction in aging, dementia, epilepsy and other brain inflammatory conditions (1).

Of note, our results showed that brain inflammation significantly increased cell proliferation, the first step of adult neurogenesis (Fig. 2). Namely, our data indicated that LPS-induced brain inflammation impaired short-term memory in spite of the fact that brain inflammation increased cell proliferation in the hippocampal dentate gyrus (Figs. 1 and 2). Similar results were also found in other studies. In brain ischemia, enhanced cell proliferation in the hippocampal dentate gyrus was observed (8) and this enhanced cell proliferation in the hippocampal dentate gyrus has been suggested as the compensatory response to excessive apoptotic cell death $(8,24)$. The role of inflammation for adult neurogenesis, however, is much more complex. Microglia activation, as an indicator of inflammation, is not pro- or anti-neurogenic per se but the net outcome is dependent on the balance between secreted molecules with pro- and anti-inflammatory action (25). In the murine experimental autoimmune encephalomyelitis model for multiple sclerosis, the most common chronic inflammatory disease of the central nervous system, proliferation regarding the hippocampal neuronal cells was increased, however hippocampal-dependent cognitive functions were massively impaired (26). Pathological conditions such as acute central nervous system insults and experimental autoimmune encephalomyelitis were accompanied by an increase in neurogenesis as well as by the induction of neurogenesis in nonneurogenic areas. These conditions might be induced by the activation of microglia and resulted in the loss of cognitive activity (27).

DCX-positive cells in the hippocampal dentate gyrus were increased in mice exhibiting autoimmune encephalomyelitis compared with those in the control group (28) DCX, a marker of newly generated neurons, is frequently regarded as an immature neuronal marker. DCX is present in the tips of neurites of non-migratory immature neurons and expressed only during three stages of neurogenesis (differentiation, migration and targeting), not the synaptic integration stage in which the newly formed cells become functionally integrated into the hippocampal network (15). In our study, the expression of DCX protein was increased through brain inflammation, indicating that brain inflammation increased the generation of immature neurons. However, both forced treadmill exercise and voluntary wheel exercise significantly suppressed the expression of DCX protein, suggesting that the generation of immature neurons by brain inflammation was inhibited by forced treadmill exercise and voluntary wheel exercise (Fig. 3).

NeuN, a specific marker of neuronal cells, recognizes nuclear proteins rather than cytoplasmic antigens or cytoplasmic organelles and does not stain the nuclei of immature nerve cells until they achieve a stage of development that approaches mature function. Thus, NeuN satisfies the primary criterion of a tissue marker regarding neuronal maturation (19). Huehnchen et al (28) also reported that despite the initial increase in the number of progenitor cells, the succeeding differentiation of BrdU-positive cells into mature NeuN-positive neurons was delayed in autoimmune encephalomyelitis mice when compared with mice in the control group. In light of the mechanism whereby cognitive deficits may be explained by altered proliferation and the differentiation of newborn hippocampal progenitors $(29,30)$, in this study, we investigated the expression of NeuN protein in the hippocampus. Brain inflammation reduced the capacity of neuronal progenitors to differentiate into mature neurons, whereas forced treadmill exercise and voluntary wheel exercise significantly increased the generation of mature neurons suppressed by brain inflammation (Fig. 4). Ekdahl et al (1) reported that the LPS-induced inflammation remarkably reduced the number of new neurons, the BrdU-immunoreactive cells double-labeled with $\mathrm{NeuN}$ in the subgranular zone of the dentate gyrus. On the other hand, physical exercise improved memory function and spatial learning ability through the enhancement of neurogenesis, which was ascertained by increasing the number of BrdU- and NeuN-double positive neurons (31).

In conclusion, the results from our study demonstrated that brain inflammation induced by LPS weakened short-term memory by increasing the generation of immature neurons and contrarily suppressed the generation of mature neurons. On the other hand, both forced treadmill exercise and voluntary wheel exercise significantly improved short-term memory by enhancing neuronal maturation. Moreover, forced treadmill exercise and voluntary wheel exercise showed similar efficacy. From these results, it can be inferred that forced treadmill exercise and voluntary wheel exercise may improve memory function deteriorated by brain inflammation, such as Alzheimer's disease and Parkinson's disease.

\section{Acknowledgements}

This study was supported by the National Research Foundation of Korea Grant funded by the Korean Government (NRF-2010327-G00127).

\section{References}

1. Ekdahl CT, Claasen JH, Bonde S, Kokaia Z and Lindvall O: Inflammation is detrimental for neurogenesis in adult brain. Proc Natl Acad Sci USA 100: 13632-13637, 2003.

2. Cacci E, Claasen JH and Kokaia Z: Microglia-derived tumor necrosis factor-alpha exaggerates death of newborn hippocampal progenitor cells in vitro. J Neurosci Res 80: 789-797, 2005.

3. Cacci E, Ajmone-Cat MA, Anelli T, Biagioni S and Minghetti L: In vitro neuronal and glial differentiation from embryonic or adult neural precursor cells are differently affected by chronic or acute activation of microglia. Glia 56: 412-425, 2008. 
4. Guo J, Li F, Wu Q, Gong Q, Lu Y and Shi J: Protective effects of icariin on brain dysfunction induced by lipopolysaccharide in rats. Phytomedicine 17: 950-955, 2010.

5. Belarbi K, Jopson T, Tweedie D, Arellano C, Luo W, Greig NH and Rosi S: TNF- $\alpha$ protein synthesis inhibitor restores neuronal function and reverses cognitive deficits induced by chronic neuroinflammation. J Neuroinflammation 9: 23, 2012.

6. Kim DH, Ko IG, Kim BK, et al: Treadmill exercise inhibits traumatic brain injury-induced hippocampal apoptosis. Physio Behav 101: 660-665, 2010.

7. Kim SE, Ko IG, Kim BK, et al: Treadmill exercise prevents aging-induced failure of memory through an increase in neurogenesis and suppression of apoptosis in rat hippocampus. Exp Gerontol 45: 357-365, 2010

8. Sim YJ, Kim SS, Kim JY, Shin MS and Kim CJ: Treadmill exercise improves short-term memory by suppressing ischemiainduced apoptosis of neuronal cells in gerbils. Neurosci Lett 372 256-261, 2004

9. Cao L, Jiao X, Zuzga DS, Liu Y, Fong DM, Young D and During MJ: VEGF links hippocampal activity with neurogenesis, learning and memory. Nat Genet 36: 827-835, 2004.

10. van Praag H, Christie BR, Sejnowski TJ and Gage FH: Running enhances neurogenesis, learning and long-term potentiation in mice. Proc Natl Acad Sci USA 96: 13427-13431, 1999.

11. Eriksson PS, Perfilieva E, Bjök-Eriksson T, Alborn AM, Nordborg C, Peterson DA and Gage FH. Neurogenesis in the adult human hippocampus. Nat Med 4: 1313-1317, 1998.

12. Kempermann G, Kuhn HG and Gage FH: More hippocampal neurons in adult mice living in an enriched environment. Nature 386: 493-495, 1997.

13. Kempermann G, Jessberger S, Steiner B and Kronenberg G: Milestones of neuronal development in the adult hippocampus. Trends Neurosci 27: 447-452, 2004

14. Ming GL and Song H: Adult neurogenesis in the mammalian central nervous system. Annu Rev Neurosci 28: 223-250, 2005.

15. von Bohlen and Halbach O: Immunohistological markers for proliferative events, gliogenesis and neurogenesis within the adult hippocampus. Cell Tissue Res 345: 1-19, 2011.

16. Francis F, Koulakoff A, Boucher D, et al: Doublecortin is a developmentally regulated, microtubule-associated protein expressed in migrating and differentiating neurons. Neuron 23: 247-256, 1999.

17. Friocourt G, Koulakoff A, Chafey P, Boucher D, Fauchereau F, Chelly J and Francis F: Doublecortin functions at the extremities of growing neuronal processes. Cereb Cortex 13: 620-626, 2003.

18. Wolf HK, Buslei R, Schmidt-Kastner R, Schmidt-Kastner PK, Pietsch T, Wiestler OD and Blümcke I: NeuN: a useful neuronal marker for diagnostic histopathology. J Histochem Cytochem 44: $1167-1171,1996$.
19. Sarnat HB, Nochlin D and Born DE: Neuronal nuclear antigen $(\mathrm{NeuN})$ : a marker of neuronal maturation in early human fetal nervous system. Brain Dev 20: 88-94, 1998.

20. Tyagi E, Agrawal R, Nath C and Shukla R: Influence of LPS-induced neuroinflammation on acetylcholinesterase activity in rat brain. J Neuroimmunol 205: 51-56, 2008.

21. van Praag H, Kempermann G and Gage FH: Running increases cell proliferation and neurogenesis in the adult mouse dentate gyrus. Nat Neurosci 2: 266-270, 1999.

22. O'Callaghan RM, Ohle R and Kelly AM: The effects of forced exercise on hippocampal plasticity in the rat: A comparison of LTP, spatial- and non-spatial learning. Behav Brain Res 176: 362-366, 2007

23. Shors TJ, Townsend DA, Zhao M, Kozorovitskiy Y and Gould E: Neurogenesis may relate to some but not all types of hippocampal-dependent learning. Hippocampus 12: 578-584, 2002.

24. Liu J, Solway K, Messing RO and Sharp FR: Increased neurogenesis in the dentate gyrus after transient global ischemia in gerbils. J Neurosci 18: 7768-7778, 1998.

25. Ekdahl CT, Kokaia Z and Lindvall O: Brain inflammation and adult neurogenesis: the dual role of microglia. Neuroscience 158 : 1021-1029, 2009.

26. Aharoni R, Arnon R and Eilam R: Neurogenesis and neuroprotection induced by peripheral immunomodulatory treatment of experimental autoimmune encephalomyelitis. J Neurosci 25: 8217-8228, 2005

27. Ziv Y, Ron N, Butovsky O, et al: Immune cells contribute to the maintenance of neurogenesis and spatial learning abilities in adulthood. Nat Neurosci 9: 268-275, 2006.

28. Huehnchen P, Prozorovski T, Klaissle P, et al: Modulation of adult hippocampal neurogenesis during myelin-directed autoimmune neuroinflammation. Glia 59: 132-142, 2011.

29. Deng W, Aimone JB and Gage FH: New neurons and new memories: how does adult hippocampal neurogenesis affect learning and memory? Nat Rev Neurosci 11: 339-350, 2010

30. Kempermann G, Gast D and Gage FH: Neuroplasticity in old age: sustained fivefold induction of hippocampal neurogenesis by long-term environmental enrichment. Ann Neurol 52: $135-143,2002$

31. Wong-Goodrich SJ, Pfau ML, Flores CT, Fraser JA, Williams CL and Jones LW: Voluntary running prevents progressive memory decline and increases adult hippocampal neurogenesis and growth factor expression after whole-brain irradiation. Cancer Res 70: 9329-9338, 2010. 